\title{
p75NTR signal transduction suppressed by BFAR and p75NTR interactions
}

\author{
LI HongMei, SHI HuiLi \& HUO KeKe* \\ State Key Laboratory of Genetic Engineering, School of Life Sciences, Fudan University, Shanghai 200433, China
}

Received October 18, 2011; accepted March 2, 2012

\begin{abstract}
p75NTR is a low-affinity nerve growth factor receptor, which promotes cell proliferation as a positive modulator of high-affinity receptor TrkA, as well as binds with cell ligands to induce apoptosis and mediate death signals. To analyze the regulatory mechanisms of p75NTR, the present study utilized a new membrane yeast two-hybrid system to screen a human fetal brain cDNA library. Results identified BFAR, a novel protein that interacts with p75NTR. Interaction specificity was verified by membrane yeast two-hybrid co-transformation assays, in vitro GST pull-down assays, and in vitro co-immunoprecipitation assays. The fluorescent subcellular localization assay revealed that the two proteins co-localized within the cytoplasm. BFAR overexpression in PC-12 and HEK293T cells inhibited the NFKB and JNK signaling pathway, as determined with the luciferase test. Co-transfected p75NTR and BFAR in HEK293T or PC-12 cells, respectively, increased the percentage of cells in the G2/M phase, decreased the number of S-phase cells, and did not change the number of G0/G1-phase cells.
\end{abstract}

p75NTR, BFAR, membrane yeast two-hybrid, protein-protein interaction, apoptosis

Citation: $\quad$ Li H M, Shi H L, Huo K K. p75NTR signal transduction suppressed by BFAR and p75NTR interactions. Sci China Life Sci, 2012, 55: 367-374, doi: $10.1007 / \mathrm{s} 11427-012-4306-\mathrm{y}$

Nerve growth factor (NGF) is a member of the neurotrophin family, which is essential for maintaining proper biological functions within the nervous system, by binding specific cell surface receptors. NGF has two structurally unrelated receptors: tyrosine kinase receptor A (TrkA), which selectively binds to NGF, and p75 neurotrophin receptor (p75NTR), which binds all members of the neurotrophin family (NGF, BDNF, NT-3, and NT-4/5). The p75NTR gene is located at $17 q 12-17 q 22$. p75NTR belongs to the type I single-pass transmembrane glycoprotein, with a molecular weight of $75 \mathrm{kD}$. This protein contains a signal peptide, an extracellular domain, a transmembrane domain, and an intracellular domain. The extracellular domain contains four highly conserved cysteine-rich domains, which serve as the ligand binding sites. The intracellular domain has at

*Corresponding author (email: kkhuo@fudan.edu.cn) least three domains - the juxtamembrane domain, the type II death domain, and the PDZ interaction domain. The juxtamembrane domain contains a 29 amino acid sequence called the "chopper domain", which has been implicated in neuronal death signaling $[1,2]$.

The p75NTR and NGF signaling pathways play an important role in nervous system development and overall health of the nervous system. However, the biological effects and regulation mechanisms of the pathways remain unclear. To detect the p75NTR signaling pathway and to analyze nervous disease mechanisms, p75NTR was utilized as a bait to screen a human embryonic brain cDNA library using the split-ubiquitin membrane yeast two-hybrid system. The assay revealed bi-functional apoptosis regulator (BFAR), a novel interaction protein of p75NTR. Results from the study revealed some biological functions of p75NTR and BFAR interactions. 


\section{Materials and methods}

\subsection{Reagents, plasmids, and antibodies}

Restriction enzymes were purchased from New England Biolabs (Ipswich, Massachusetts, USA); T4 DNA ligase and DNA marker were purchased from TaKaRa (Shiga, Japan); Pfu enzyme, Taq enzyme, and ssDNA were products of Beijing BioDev-Tech (Beijing, China); PCR product clean up kit, DNA fragment gel extraction kit, and plasmid extraction kit were purchased from Axygen (Hangzhou, China); 3-AT was purchased from Sigma \& Aldrich (St. Louis, Missouri, USA); DUAL membrane yeast two-hybrid system kit, human embryonic brain cDNA library, and the HTX $\beta$-galactosidase assay kit were purchased from Dualsystems Biotech (Schlieren, Switzerland); Isopropyl $\beta$-D-1-Thiogalactopyranoside (IPTG), protease inhibitor cocktails, and Phenylmethanesulfonyl fluoride (PMSF) were products of Amresco (Solon, Ohio, USA); Ni-NTA agarose beads were purchased from Qiagen (Hilden, Germany); glutathione sepharose $4 \mathrm{~B}$ beads were purchased from Pharmacia Biotech (Stockholm, Sweden).

DMEM high-glucose medium and fetal bovine serum for cell culture were products of Hyclone (Logan, Utah, USA); trypsin-EDTA and opti-DMEM were purchased from Gibco (Langley, Oklahoma, USA); Lipofectamin ${ }^{\mathrm{TM}} 2000$ was purchased from Invitrogen (Carlsbad, California, USA); protein $\mathrm{A}+\mathrm{G}$ beads and mouse monoclonal antibody against Myc and Flag were purchased from Sigma \& Aldrich; rabbit monoclonal antibody against p75NTR was purchased from Abcam (Cambridge, England); goat anti-rabbit secondary antibody-conjugated HRP and rabbit anti-mouse red secondary antibody-conjugated $\mathrm{Cy} 3$ were products of ProteinTech (Chicago, Illinois, USA); rabbit anti-mouse secondary antibody-conjugated HRP was from Rockland (Gilbertsville, Pennsylvania, USA); SuperSigna West Femto Maximum Sensitivity Substrate was purchased from Pierce (Rockford, Illinois, USA); RNase A was the product of Beijing JingKeHongDa Biotechnology (Beijing, China); propidium iodide (PI) was purchased from KeyGEN Biotech (Shanghai, China); dual luciferase reporter assay system was the product of Promega (Fitchburg, Wisconsin, USA).

The vector pMT18-T used for plasmid construction was from TaKaRa; the prokaryotic cell expression vector pGEX-5X-1 and pET-28a were from Pharmacia Biotech and Novagen (Darmstadt, Germany), respectively; the animal expression vector $\mathrm{pEF}-\mathrm{Flag}$ and $\mathrm{pCMV-Myc}$ were from Invitrogen, and pEGFP-C1 was from Clontech (Mountain View, California, USA). The E. coli strains TOP10, BL21, and BL21 (DE3), as well as the animal cell lines HEK293T, HeLa and PC-12 were maintained in our laboratory.

\subsection{Gene cloning of p75NTR}

According to the gene annotation from Ensembl and Gen-
Bank (accession number: NM_002507), the entire p75NTR gene length was $3417 \mathrm{bp}$ and the CDS length was $1284 \mathrm{bp}$, respectively, encoding 427 amino acids, with a total of six exons. The first exon was a signal peptide that contained 66 amino acids. The $p 75 N T R$ gene was cloned from human hepatoma cell line SMMC7721 genomic DNA by amplifying six exons by PCR. Full-length p75NTR was obtained by splicing the exons. The amplification primer sequences were as follows: Exon1 F: 5'-AGAGGCCATTACGG CCATGGGGGCAGGTGCCAC-3', Exon1 R: 5'-GCACT CACCGCTGTGTGTGTACAG-3'; Exon2 F: 5'-CAAGG CCATTACGGCCAAGGAGGCATGCCCCAC-3', Exon2 R: 5'-CAGAGAATGTCACACTGTCCAGGCAGGGCTC3'; Exon3 F: 5'-AGTGTGACATTCTCTGACGTGGTGA3', Exon3 R: 5'-CCTCGCACTCGGCGTCGG-3'; Exon4 F: 5'-CCGACGCCGAGTGCGAGGAGATCCCTGGCCGTT G-3', Exon4 R: 5'-TGTTCCACCTCTTGAAGGCTATGT AG-3'; Exon5 F: 5'-TCGAATTCAAGAGGTGGAACAGC TGCAAGCAGAA-3', Exon5 R: 5'-CTTGAGGGCCTGT CCCGAGGCTGTCT-3'; Exon6 F: 5'-GGACAGGCCCTC AAGGGTGACG-3', Exon6 R: 5'-AGAGGCCGAGGCGG CCTCACACCGGGGATGTGG-3'.

\subsection{Screening the interaction protein of p75NTR by membrane yeast two-hybrid}

Membrane yeast two-hybrid screen assay was performed according to DUAL membrane system manufacturer protocols (Dualsystems Biotech, Switzerland). The system required that Cub-LexA-VP16-bait and NubG-prey were located in the cytosol. Therefore, the $p 75 N T R$ gene was subcloned into the correct bait vector. To determine whether pBT3-SUC-p75NTR was the appropriate bait, a self-activation and functional assay were performed. The screening conditions were then optimized in a pilot screening, and the bait pBT3-SUC-p75NTR was screened against the human embryonic brain NubG-fused cDNA library.

\subsection{GST pull-down assay}

p75NTR was cloned into the expression vector pGEX-5X-1 and transformed into the $E$. coli strain BL21. The cells were incubated in $100 \mu \mathrm{mol} \mathrm{L}{ }^{-1}$ IPTG for $2 \mathrm{~h}$ at $28^{\circ} \mathrm{C}$, followed by centrifugation and re-suspension in $2 \mathrm{~mL}$ PBS. Following addition of PMSF and protease inhibitors, the cells were sonicated, followed by incubation with $100 \mu \mathrm{L} 20 \%$ Triton $\mathrm{X}-100$ on ice for $30 \mathrm{~min}$. The protein was then purified through glutathione-sepharose 4B beads (Invitrogen). Full-length BFAR was cloned into the vector pET-28a and expressed in the $E$. coli strain BL21 (DE3), followed by incubation with $100 \mu \mathrm{mol} \mathrm{L}{ }^{-1}$ IPTG for $6 \mathrm{~h}$ at $22^{\circ} \mathrm{C}$. After the cells were collected by centrifugation and re-suspended in $2 \mathrm{~mL} \mathrm{Ni}$-lysis buffer $\left(50 \mathrm{mmol} \mathrm{L}^{-1}\right.$ sodium phosphate, $300 \mathrm{mmol} \mathrm{L}^{-1} \mathrm{NaCl}, 10 \mathrm{mmol} \mathrm{L}^{-1}$ imidazole, $\mathrm{pH} \mathrm{8.0)}$, the 
cells were sonicated and purified by Ni-NTA agarose (Qiagen), the beads were washed three times with $200 \mu \mathrm{L}$ wash buffer $\left(50 \mathrm{mmol} \mathrm{L}^{-1}\right.$ sodium phosphate, $300 \mathrm{mmol} \mathrm{L}^{-1} \mathrm{NaCl}$, $20 \mathrm{mmol} \mathrm{L}^{-1}$ imidazole, $\mathrm{pH} 8.0$ ), and the protein was eluted with $60 \mu \mathrm{L}$ elution buffer $\left(50 \mathrm{mmol} \mathrm{L}{ }^{-1}\right.$ sodium phosphate, $300 \mathrm{mmol} \mathrm{L}^{-1} \mathrm{NaCl}, 250 \mathrm{mmol} \mathrm{L}^{-1}$ imidazole, $\mathrm{pH}$ 8.0). The two purified proteins were incubated in $500 \mu \mathrm{L}$ NETN buffer $\left(100 \mathrm{mmol} \mathrm{L}^{-1} \mathrm{NaCl}, 1 \mathrm{mmol} \mathrm{L}{ }^{-1}\right.$ EDTA, $20 \mathrm{mmol}$ $\mathrm{L}^{-1}$ Tris- $\mathrm{HCl}, 0.5 \%$ Nonidet P-40, $1 \mathrm{mmol} \mathrm{L}^{-1} \mathrm{PMSF}, \mathrm{pH}$ 7.0) at $4{ }^{\circ} \mathrm{C}$ for $4-8 \mathrm{~h}$, and the beads washed three times with buffer $\mathrm{H}\left(20 \mathrm{mmol} \mathrm{L}^{-1}\right.$ HEPES, $50 \mathrm{mmol} \mathrm{L}^{-1} \mathrm{KCl}, 20 \%$ glycerol, $0.1 \%$ Nonidet P-40, $0.007 \%$-mercaptoethanol, $\mathrm{pH}$ 7.7). The protein-bound beads were subjected to Western blot analysis with mouse anti-His antibody (Sigma).

\subsection{Co-immunoprecipitation assay}

p75NTR was cloned into the vector pEF-Flag, and full-length BFAR was cloned into the vector pCMV-Myc. The two plasmids were co-transfected into $\mathrm{PC}-12$ cells with Lipofectamin 2000 . The cells were incubated at $37^{\circ} \mathrm{C}$ in $5 \%$ $\mathrm{CO}_{2}$ for $36-48 \mathrm{~h}$, then collected and washed in ice-cold PBS, followed by the addition of $1 \mathrm{~mL}$ lysis buffer. A total of 40 $\mu \mathrm{L}$ lysis supernatant was used for Western blot analysis of plasmid expression level. The cell lysates were pre-cleared with protein $\mathrm{A} / \mathrm{G}$ agarose beads for $1 \mathrm{~h}$ at $4^{\circ} \mathrm{C}$, then immunoprecipitated with $2 \mu \mathrm{g}$ relative antibody at $4^{\circ} \mathrm{C}$ for $1 \mathrm{~h}$ and incubated in $20 \mu \mathrm{L}$ protein $\mathrm{A} / \mathrm{G}$ agarose at $4^{\circ} \mathrm{C}$ overnight. The immunoprecipitated agarose was washed three times in ice-cold PBS, then subjected to SDS-PAGE and Western blot analysis using anti-Myc or anti-p75NTR antibodies.

\subsection{Immunofluorescence imaging of HeLa cells}

Full-length BFAR was cloned into the vector pEGFP-C1 and transiently co-transfected into $\mathrm{HeLa}$ cells with pEF-Flag-p75NTR. Following incubation at $37^{\circ} \mathrm{C}$ in $5 \%$ $\mathrm{CO}_{2}$ for $24-36 \mathrm{~h}$, the cells were fixed in $4 \%$ paraformaldehyde for $1 \mathrm{~h}$, permeabilized in $1 \%$ Triton $\mathrm{X}-100$ for $10 \mathrm{~min}$, blocked in $1 \%$ bovine serum albumin for $1 \mathrm{~h}$, and incubated in anti-p75NTR antibody used at a $1 / 200$ dilution at $4^{\circ} \mathrm{C}$ overnight. The cells were then washed five times in PBST, incubated in goat anti-rabbit-Cy3 antibody at a 1/500 dilution for $1 \mathrm{~h}$, washed five times with PBST, and stained with $1 \mu \mathrm{g} \mathrm{mL}{ }^{-1}$ Hoechst 33542 for $10 \mathrm{~min}$. The cells were then analyzed by fluorescence microscopy (Zeiss) (green fluorescent at $480 \mathrm{~nm}$ and red fluorescence at $568 \mathrm{~nm}$ ).

\subsection{Luciferase activity assay}

Cells were digested in $0.05 \%$ trypsin and cultured in 24 -well plates at a density of $2 \times 10^{5}$ cells $\mathrm{mL}^{-1}$ one day prior to transfection. The signal pathway reporter plasmid (p53-luc, NFkB-luc, CRE-luc, SRE-luc, or AP1-luc), internal reference plasmid Rellina, and the plasmid of interest or empty control plasmid were co-transfected into the cells. Following incubation at $37^{\circ} \mathrm{C}$ in $5 \% \mathrm{CO}_{2}$ for $48 \mathrm{~h}$, the cells were lysed in Passive Lysis Buffer (PLB) according to the Promega dual luciferase reporter assay system protocol. Luciferase activity was measured by luminescence. Each test required $5 \mu \mathrm{L}$ of cell lysis supernatant, with $25 \mu \mathrm{L}$ LARII to determine firefly luciferase activity, and $25 \mu \mathrm{L}$ Stop \& Glo substrate solution for Rellina luciferase activity. Each sample was analyzed in triplicate.

\subsection{Cell cycle assay}

The cells were digested in $0.05 \%$ trypsin, washed two times in PBS, fixed in ice-cold $75 \%$ ethanol at $4{ }^{\circ} \mathrm{C}$ overnight, and washed two times in PBS. The cells were then re-suspended in $400 \mu \mathrm{L}$ PBS and incubated in $4 \mu \mathrm{L} \mathrm{PI}\left(1 \mathrm{mg} \mathrm{mL}^{-1}\right)$ and 10 $\mu \mathrm{L}$ RNase A (10 $\mathrm{mg} \mathrm{mL}^{-1}$ ) in darkness for $10 \mathrm{~min}$ at room temperature. The cells were then filtered through a $200 \mu \mathrm{m}$ mesh nylon membrane, and cell cycle analysis was analyzed by FACS. Each experiment was repeated in triplicate.

\section{Results}

\subsection{Identification of potential interactional proteins of p75NTR using the membrane yeast two-hybrid system}

Because p75NTR was a type-I membrane protein, the dual membrane yeast two-hybrid system was utilized to identify further interactional proteins. For the self-activation and functional assay, the bait pBT3-SUC-p75NTR was co-transformed with pOst1-NubI and the empty library vector pPR3-N into the yeast strain NMY51, respectively. The transformed yeast cells were streaked onto SD-trp-leu (SD-TL), SD-trp-leu-his (SD-TLH), and SD-trp-leu-his-ade (SD-TLHA) selection plates and incubated for $3 \mathrm{~d}$ at $30^{\circ} \mathrm{C}$, respectively. pTSU2-APP/ pNubG-Fe65 was used as a positive control and pTSU2- APP/pPR3-N as a negative control. Results showed that the co-transformation of p75NTR with pPR3-N did not grow on SD-TLH and SD-TLHA selection plates, demonstrating that p75NTR exhibited no self-activation activity (Table 1). However, co-transformation of p75NTR and pOst1-NubI did grow on SD-TLH and SD-TLHA selection plates, suggesting that HIS3 and ADE2 reporter genes were activated (Table 1). Subsequently, a human fetal brain cDNA library was transformed into NMY51-expressing p75NTR, and the interactors were selected using stringency conditions on SD-TLHA containing $1 \mathrm{mmol} \mathrm{L}{ }^{-1} 3$-AT plates. A total of 89 positive clones were obtained from $3.9 \times 10^{5}$ transformants, all of which belonged to 38 genes, according to BLAST analysis. One of the positive clones encoded BFAR (bifunctional apoptosis regulator). To confirm the interaction between p75NTR and 
Table 1 p75NTR functional assay results, p75NTR self-activation and functional assay results. Data represent clone growth on the selection plate

\begin{tabular}{cccc}
\hline Self-activation and functional assay & SD-TL & SD-TLH & SD-TLHA \\
\hline pBT3-SUC-p75NTR/pOst1-NubI & 810 & 151 & 64 \\
pBT3-SUC-p75NTR/pPR3-N & 809 & 1 & 2 \\
PTSU2-APP/pNubG-Fe65(+) & 670 & 400 & 350 \\
PTSU2-APP/pPR3-N(-) & 650 & 2 & 0 \\
\hline
\end{tabular}

BFAR, full-length BFAR cDNA was cloned into the prey vector pPR3-N and co-transformed with pBT3-SUCp75NTR into NMY51 strain cells. The resulting co-transformant was assayed for growth on the same selective medium that was used in the library screen, as well as for color formation in the $\beta$-galactosidase assay. Results showed that all reporter genes were active, but the negative control did not exhibit $\beta$-galactosidase activity (Figure 1).

\section{2 p75NTR interacts with BFAR in vitro}

To exclude false positives in the yeast cultures, GST pulldown assays were employed to verify interactions between p75NTR and BFAR. Results showed that the GST protein did not bind to BFAR (Figure 2, line 2), but the GST-

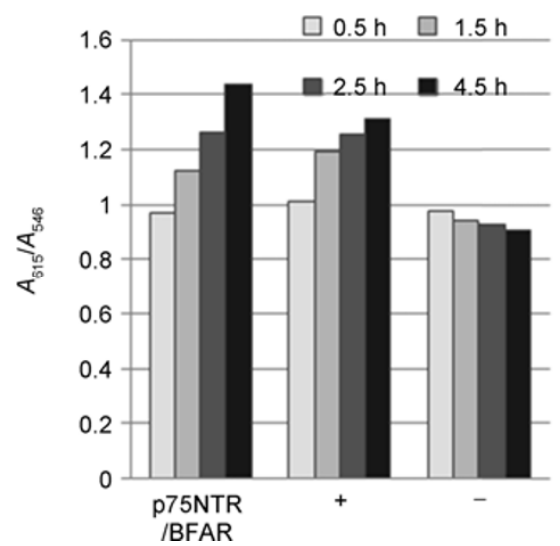

Figure 1 Yeast two-hybrid interactions between p75NTR and BFAR. pBT-SUC-p75NTR/pPR3-N-BFAR were co-transformed into the yeast strain NMY51, and the HTX $\beta$-galactosidase assay was performed to measure reaction absorbance at $0.5,1.5,2.5$, and $4.5 \mathrm{~h}$. An $A_{615} / A_{546}$ ratio $>1.0$ represents lacZ activity, but $<1.0$ represents inactivity. "+”, positive control pTSU2-APP/pNubG-Fe65; “-”, negative control pBT3-SUCp75NTR/pPR3-N.

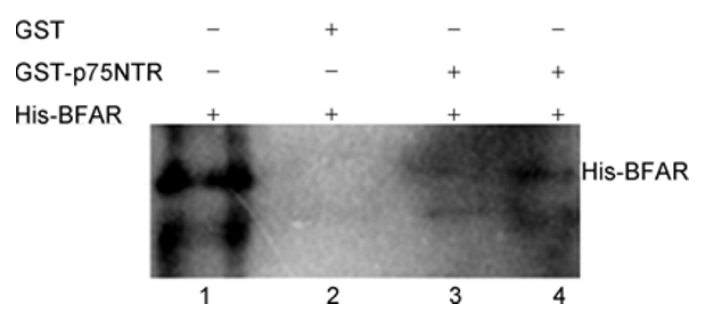

Figure 2 In vitro GST pull-down assay. Line 1, pET-28a-BFAR input; line 2, pGEX-5X-1 empty vector and pET-28a-BFAR expression; lines 3 and 4, pGEX-5X-1-p75NTR and pET-28a-BFAR expression, respectively.
p75NTR protein bound to the BFAR protein in vitro (Figure 2 , lines 3 and 4), demonstrating that the two proteins directly interacted in vitro.

\section{3 p75NTR interacts with BFAR in vitro}

To assess whether p75NTR interacted with BFAR, PC-12 cells were transiently co-transfected with pEF-Flagp75NTR and pCMV-Myc-BFAR, or with pEF-Flagp75NTR and pCMV-Myc as the negative controls, respectively. Following 48-h incubation, whole lysates were subjected to immunoprecipitation with anti-p75NTR or anti-Myc antibody. Results showed that p75NTR was recognized by the Myc-BFAR immunoprecipitates (Figure 3, line 2 ), but Myc was not recognized (Figure 3, line 1), which suggested that p75NTR specifically co-immunoprecipitated with BFAR.

\section{4 p75NTR and BFAR co-localized in HeLa cells}

To further examine the interaction between p75NTR and BFAR in living cells, pEGFP-C1-BFAR and pEFFlag-p75NTR were transfected, respectively, or co-transfected into HeLa cells, followed by immunofluorescence co-localization using confocal fluorescence microscopy. Results demonstrated that BFAR was localized in the cytoplasm (green) when expressed alone, and p75NTR was expressed in the cell cytoplasm and cell membrane (red). However, when co-expressed, p75NTR and BFAR colocalized to the cytoplasm (orange) (Figure 4). These results suggested that p75NTR physically interacted with BFAR in vitro.

\section{5 p75NTR and BFAR interaction effects on signal pathway}

p75NTR-mediated apoptosis has been widely reported $[3,4]$.

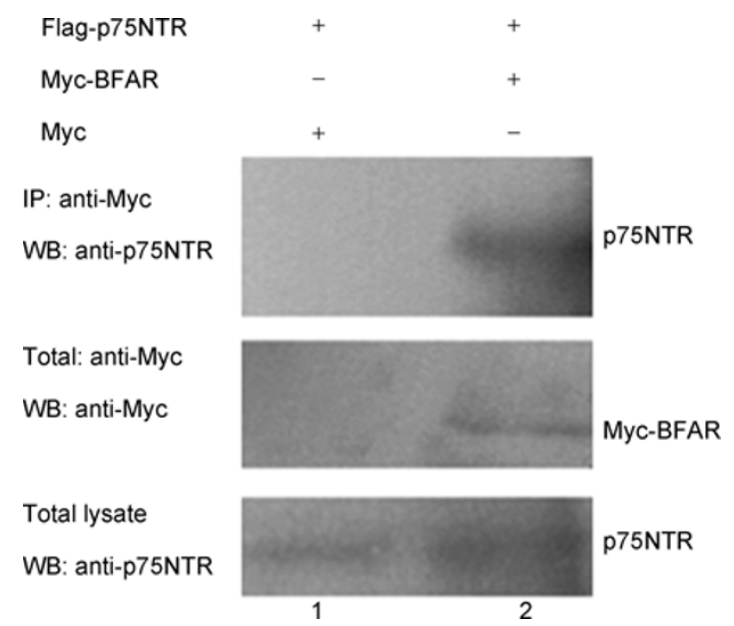

Figure 3 Co-immunoprecipitate assay in vitro. Line 1, pEF-Flagp75NTR and pCMV-Myc co-transfected into PC-12 cells; line 2, pEFFlag-p75NTR and pCMV-Myc-BFAR co-transfected into PC-12 cells. 


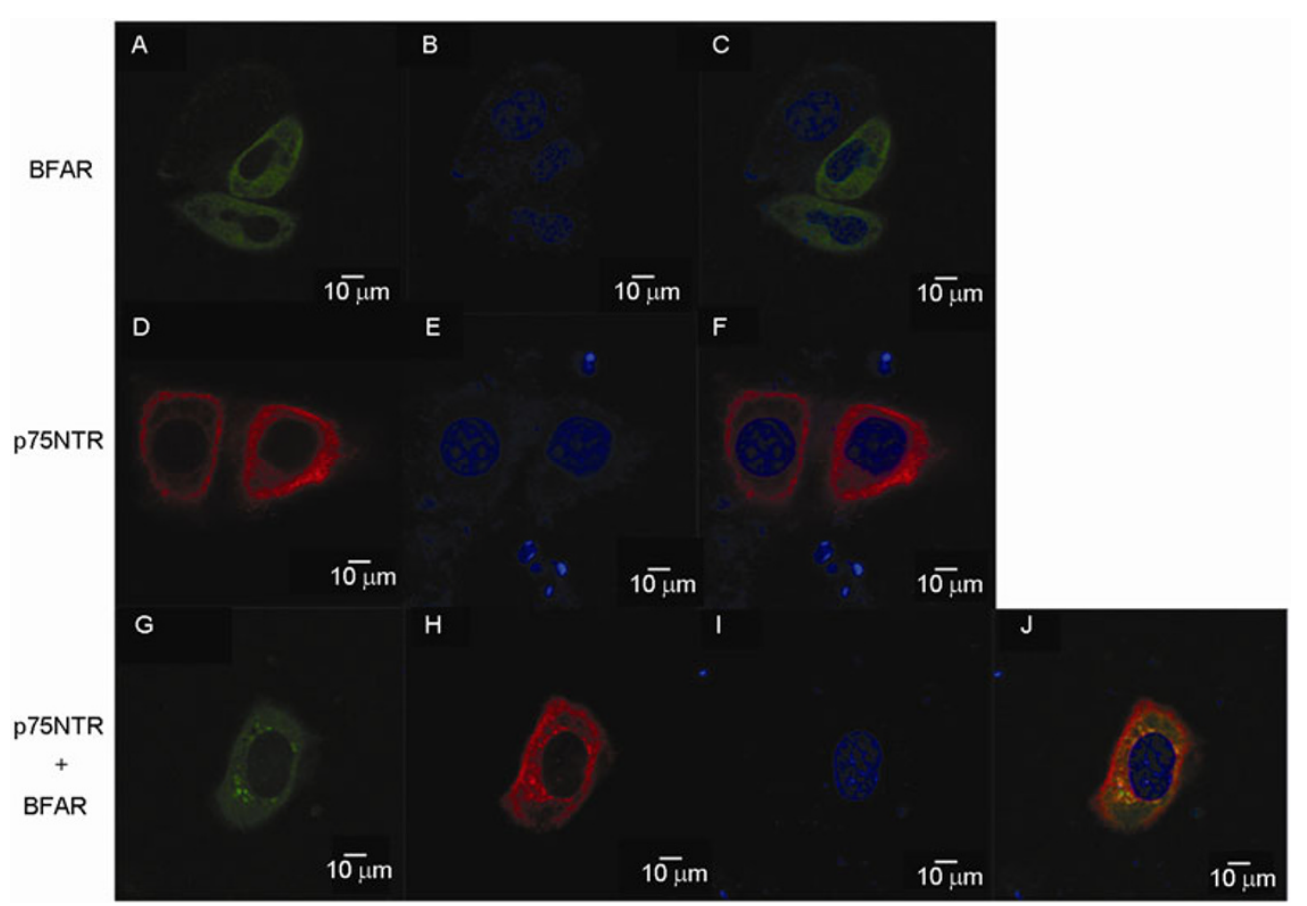

Figure 4 Co-localization of p75NTR and BFAR in HeLa cells. A-C, pEGFP-C1-BFAR transfected into HeLa cells alone. D-F, pEF-Flag-p75NTR transfected into HeLa cells alone; cells were analyzed using immunofluorescence. G-J, pEF-Flag-p75NTR and pEGFP-C1-BFAR co-transfected into HeLa cells. The right images (C, F, and J) show merged images from the two left images, respectively.

In addition, p75NTR plays an important role in the regulation of cell cycle and $\beta$-amyloid formation [5,6]. Previous studies have shown that NFאB, JNK, p53, ceramide, and other molecules are involved in p75NTR apoptotic signal transduction [7-11]. BFAR is a dual-functional apoptosis regulator [12]. To determine whether interactions between BFAR and p75NTR affected p75NTR-mediated apoptosis signal transduction, the dual luciferase assay system (Promega) was used to detect the effect of BFAR on four reporter genes from the NFKB and JNK signaling pathways in PC-12 and HEK293T cells, respectively (Table 2). pCMV-Myc-BFAR or empty vector pCMV-Myc, reporter plasmid, and TP-Rellina were co-transfected into PC-12 cells. Following 48-h incubation, the cells were lysed with PLB, and lysis supernatant luciferase activity was measured by luminescence. Results demonstrated that BFAR suppressed $N F \kappa B, A P 1, S R E$, and $C R E$ reporter gene transcriptional activity in PC-12 cells. Compared with the empty plasmid group, all four signaling pathways were inhibited after transfection with pCMV-Myc-BFAR, and luciferase activity decreased by $48.67 \%, 31.41 \%, 22.19 \%$, and $28.04 \%$, respectively (Figure 5A). In contrast, pCMV-Myc-BFAR and pEF-Flag-p75NTR were co-transfected into HEK293T cells, meanwhile, the empty vector pCMV-Myc were co-transfected with pEF-Flag-p75NTR as the negative controls, demonstrating that BFAR and p75NTR inhibited $\mathrm{NF} \mathrm{B}, \mathrm{AP} 1, \mathrm{SRE}$, and CRE activity to varying degrees in HEK293T cells, respectively (Figure 5B).

\subsection{BFAR over-expression affects the cell cycle}

Suppression of p75NTR expression in PC-12 cells has been shown to induce up-regulation of cyclin D2 [5]. In addition, over-expression of BFAR impacts multiple signaling pathways in PC-12 cells to varying degrees. To determine whether BFAR affects the cell cycle, PC-12 cells were transiently transfected with pCMV-Myc-BFAR or pCMV-Myc, respectively. As shown in Figure 6A, the percentage of cells at $\mathrm{S}$ phase was less in BFAR-PC-12 cells $(17.15 \%)$ than in Vec-PC-12 cells $(25.57 \%)$, while the percentage of cells at the $\mathrm{G} 2 / \mathrm{M}$ phase was greater in BFAR-PC-12 cells (14.82\%) than in Vec-PC-12 cells $(10.49 \%)$. However, the percentage of cells at the G0/G1 phase remained unchanged $(68.05 \%$ in BFAR-PC-12 cells and $64.74 \%$ in Vec-PC-12 cells). Similar results were also demonstrated in BFAR-HEK293T cells, as shown in Figure 6B. The percentage of cells in the $S$ phase was less in BFAR-HEK293T cells $(28.71 \%)$ than in Vec-HEK293T cells (37.85\%), while the percentage of cells in the G2/M phase was greater in BFAR-HEK293T cells

Table 2 Cell signal pathway information

\begin{tabular}{|c|c|c|c|}
\hline $\begin{array}{c}\text { cis-acting } \\
\text { Enhancer element }\end{array}$ & Abbreviation & $\begin{array}{l}\text { Transcription } \\
\text { factor(s) }\end{array}$ & $\begin{array}{c}\text { Signal transduction } \\
\text { pathways }\end{array}$ \\
\hline Activator Protein 1 & AP1 & c-jun/c-fos & JNK \\
\hline Nuclear factor of $\kappa \mathrm{B}$ cells & NFкB & NFкB & NFKB \\
\hline Serum response element & SRE & Elk-1/SRF & MAPK/JNK \\
\hline cAMP response element & CRE & ATF2/CREB & $\mathrm{JNK} / \mathrm{p} 38 \& \mathrm{PKA}$ \\
\hline
\end{tabular}



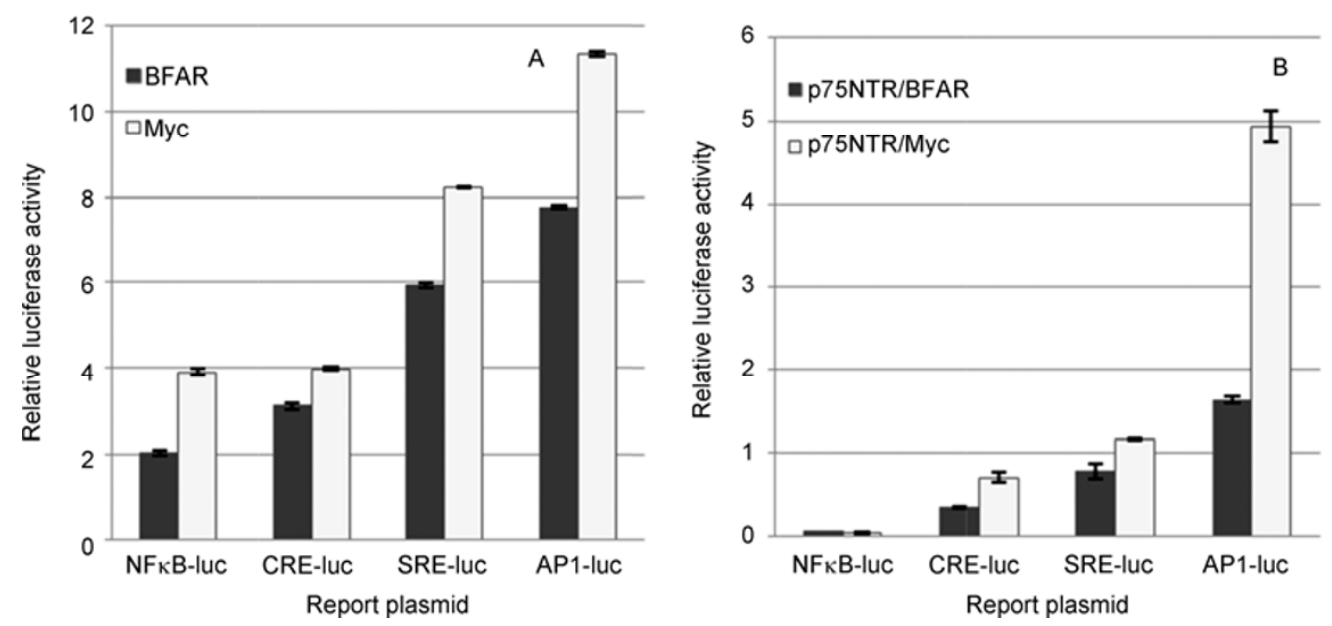

Figure 5 BFAR inhibits NFאB, AP1, SRE, and CRE signal pathway activity. A, BFAR individually inhibits all four signal pathways in PC-12 cells. B, Co-transfected p75NTR and BFAR inhibit activity in all four signal pathways in HEK293T cells.
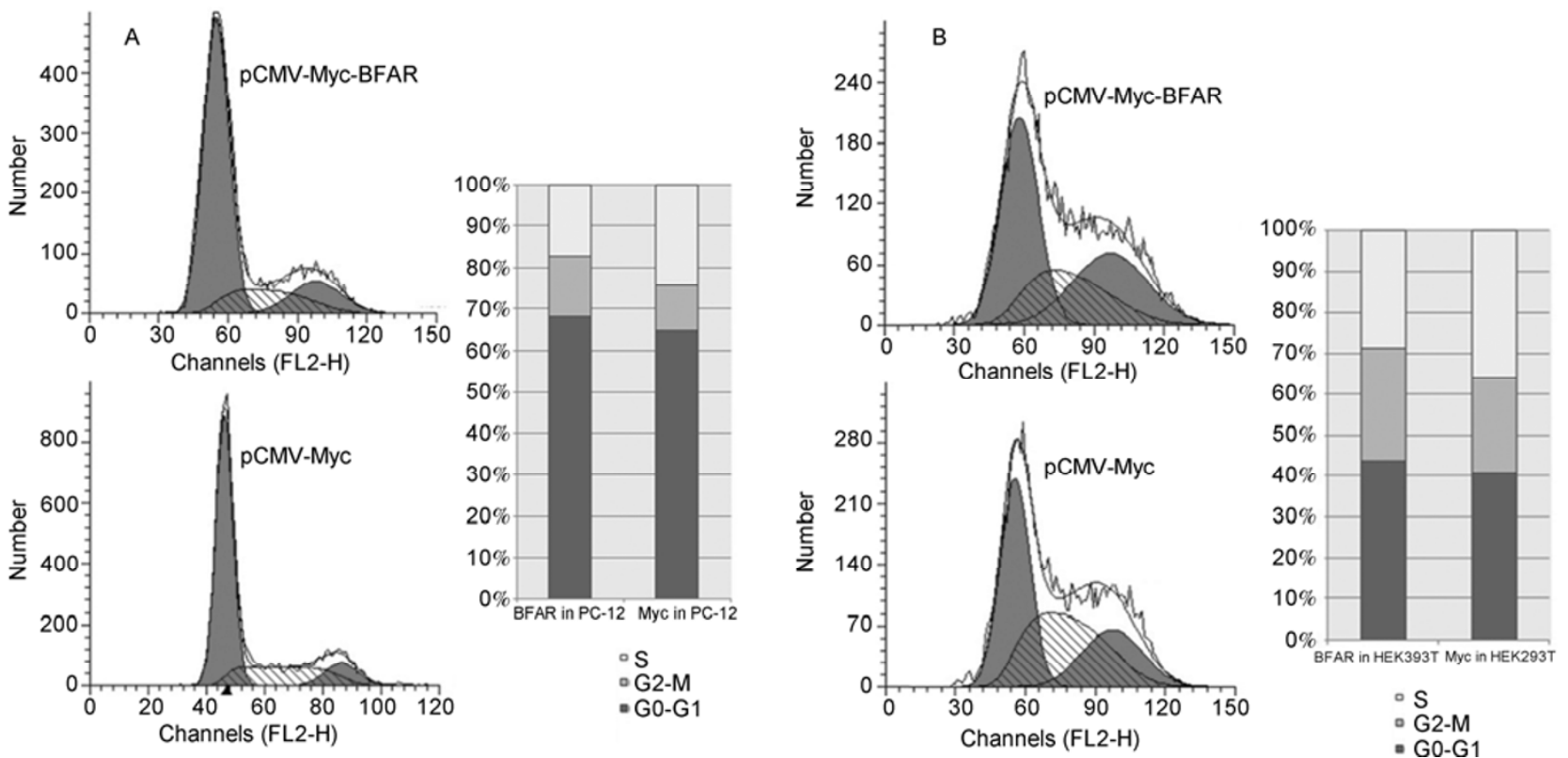

Figure 6 BFAR over-expression affects cell cycle, as determined by flow cytometry. A, pCMV-Myc-BFAR and pCMV-Myc were transfected into PC-12 cells, respectively. B, pCMV-Myc-BFAR and pCMV-Myc were transfected into HEK293T cells, respectively.

(28.82\%) than in Vec-HEK293T cells (22.19\%). In addition, the percentage of cells in the G0/G1 phase was $42.47 \%$ and $49.96 \%$, respectively. These results suggested that BFAR over-expression significantly affected cell proliferation.

\section{Discussion}

As a transmembrane protein, the low-affinity receptor p75NTR is widely expressed in tissues and tumor cells of the nervous and non-nervous systems, and is also up-regulated following injury. p75NTR promotes cell survival as a co-receptor for TrkA-mediated death signals, and p75NTR death signaling closely correlates with a number of human neurodegenerative diseases, such as Alzheimer's disease and motor neuron disease $[1,2,13,14]$. Therefore, there is a need for a better understanding of p75NTR protein interactions, as well as the physiological and pathological molecular mechanisms of action.

Yeast two-hybrid technology is an efficient method for studying protein-protein interactions within living cells, and a number of proteins have been identified to interact with p75NTR using this technology [15-18]. However, the conventional yeast two-hybrid system has limitations for studying membrane-protein interactions. First, the conventional two-hybrid assay requires that bait and prey proteins must be targeted to the nucleus. Therefore, only the intracellular domain of membrane proteins can be identified using this 
approach. Second, the captured target proteins are primarily intracellular soluble proteins, rather than membrane-associated proteins.

The DUAL membrane system is a yeast-based screening assay, which takes advantage of the split-ubiquitin system developed by Johnsson and Varshavsky to detect interactions between integral membrane proteins, membrane-associated proteins, and soluble proteins in their natural setting [19]. In the present study, the DUAL membrane system was utilized to screen a human fetal brain cDNA library with p75NTR as the bait to identify the interacting partner BFAR. The protein interaction was further confirmed using the GST pull-down in vitro assay, as well as in vitro co-immunoprecipitation, and subcellular co-localization experiments.

The BFAR gene encodes a protein composed of 450 amino acids, which contains four recognizable domains [20,21]. These domains include an N-terminal zinc-binding RING domain for binding ubiquitin conjugating enzymes (E2s), a SAM domain that interacts with Bcl-2 and Bcl-XL and suppresses Bax-induced cell death, a DED-like domain for blocking activation of the Fas-induced apoptosis pathway, and a C-terminal transmembrane (TM) domain that enables BFAR to insert into the endoplasmic reticulum membrane. Immunoblot analysis of normal human tissues has shown that BFAR is highly expressed in the brain [12]. In addition, endogenous BFAR expression is reduced under prolonged ER stress [22].

p75NTR and BFAR are integrated membrane proteins. p75NTR is synthesized in the endoplasmic reticulum and then transferred to the plasma membrane, while BFAR is primarily localized in the endoplasmic reticulum [12]. Therefore, the two proteins are in close proximity to each other, allowing for interactions within the endoplasmic reticulum or cytoplasm, which was confirmed by immunofluorescence analysis from the present study. It has been reported that HIP1, Hippi, and Bap31 all contain a pseudo-DED domain, which interacts with BFAR [12]. p75NTR also contains an analogous DD domain, which might be the structure that mediates interactions between BFAR and p75NTR.

Previous studies have shown that NFאB and JNK signaling correlate with p75NTR-mediated apoptosis [7-11,23,24]. In addition, BFAR has been shown to suppress cell apoptosis, although p75NTR, as a NGF receptor, induces cell apoptosis $[25,26]$. The functional antagonism could indicate that BFAR inhibited p75NTR-mediated cell apoptosis. In the present study, p75NTR and BFAR co-transfection into PC-12 or HEK293T cells resulted in inhibition of NFKBand JNK-related signal pathways, as determined by the luciferase reporter test. However, the physiological significance of BFAR inhibition on these signaling pathways remains to be shown.

Although the pathological features of neurodegenerative diseases have been well-studied, effective therapeutics re- main limited, primarily because the regulatory mechanisms of neuronal cell survival and death remain unclear. In summary, results from the present study demonstrated specific protein-protein interactions between $\mathrm{p} 75 \mathrm{NTR}$ and BFAR, as well as interaction effects on the p75NTR-mediated signaling pathway and cell cycle in PC-12 and HEK293T cells. Future studies of the effects of p75NTR and BFAR interactions on neuroprotection regulation are necessary to better understand neuronal survival and death regulatory mechanisms, as well as to provide new methods for treating neurodegenerative diseases.

We thank Prof. Shen AiGuo and Dr. Tao Tao for helpful discussion. We thank Dr. Luo ShiPing for technical assistance. This work was supported by the National High Technology Research and Development Program of China (Grant No. 2006AA02A310), National Science and Technology Key Program of China (Grant Nos. 2008ZX10003-006 and 2009ZX09301011) and National Basic Research Program of China (Grant No. 2010CB912609) which were awarded to Huo KeKe.

1 Coulson E J, Reid K, Baca M, et al. Chopper, a new death domain of the p75 neurotrophin receptor that mediates rapid neuronal cell death. J Biol Chem, 2000, 275: 30537-30545

2 Clare K U, Elizabeth J C. The p75 neurotrophin receptor. Int J Biochem Cell Biol, 2008, 40: 1664-1668

3 Rabizadeh S, Oh J, Zhong L, et al. Induction of Apoptosis by the low-affinity NGF receptor. Science, 1993, 261: 345-348

4 Salehi A H, Roux P P, Kubu C J, et al. NRAGE, a novel MAGE protein, interacts with the $\mathrm{p} 75$ neurotrophin receptor and facilitates nerve growth factor-dependent apoptosis. Neuron, 2000, 27: 279-288

5 Fritz M D, Mirnics Z K, Nylander K D, et al. p75NTR enhances PC-12 cell tumor growth by a non-receptor mechanism involving downregulation of cyclin D2. Exp Cell Res, 2006, 312: 3287-3297

6 Wang Y J, Wang X, Lu J J, et al. p75NTR regulates Abeta deposition by increasing Abeta production but inhibiting Abeta aggregation with its extracellular domain. J Neurosci, 2011, 31: 2292-2304

7 Carter B D, Kaltschmidt C, Kaltschmidt B, et al. Selective activation of NF-kappa B by nerve growth factor through the neurotrophin receptor p75. Science, 1996, 272: 542-545

8 Khursigara G, Orlinick J R, Chao M V. Association of the p75 neurotrophin receptor with TRAF6. J Biol Chem, 1999, 274: 2597-2600

9 Bhakar A L, Howell J L, Paul C E, et al. Apoptosis induced by p75NTR overexpression requires Jun kinase-dependent phosphorylation of Bad. J Neurosci, 2003, 23: 11373-11381

10 Yoon S O, Bonnefil C P, Carter B, et al. Competitive signaling between TrkA and p75 nerve growth factor receptors determines cell survival. J Neurosci, 1998, 18: 3273-3281

11 Gentry J J, Bonnefil C P, Carter B D. Nerve growth factor activation of nuclear factor kappa B through its p75 receptor is an anti-apoptotic signal in RN22 schwannoma cells. J Biol Chem, 2000, 275: 7558-7565

12 Roth W, Kermer P, Krajewskal M, et al. Bifunctional apoptosis inhibitor (BAR) protects neurons from diverse cell death pathways. Cell Death Differ, 2003, 10: 1178-1187

13 Dechant G, Barde Y A. The neurotrophin receptor p75NTR: Novel functions and implications for diseases of the nervous system. Nat Neurosci, 2002, 5: 1131-1136

14 José M F, Noelia L S. A novel hypothesis for Alzheimer disease based on neuronal tetraploidy induced by p75NTR. Cell Cycle, 2010, 9: 1934-1941

15 Mukai J, Hachiya $\mathrm{T}$, Hoshino S S, et al. NADE, a p75NTRassociated cell death executor, is involved in signal transduction mediated by the common neurotrophin receptor p75NTR. J Biol Chem, 2000, 275: 17566-17570 
16 Bai D, Chen H, Huang B. RanBPM is a novel binding protein for p75NTR. Biochem Biophys Res Commun, 2003, 309: 552-557

17 Nichols A, Martinou I, Maundrell K, et al. The p75 neurotrophin receptor: effects on neuron survival in vitro and interaction with death domain-containing adaptor proteins. Apoptosis, 1998, 3: 289294

18 Roxana P, Melinda B, James D D, et al. Sall2 is a novel p75NTR-interacting protein that links NGF signalling to cell cycle progression and neurite outgrowth. EMBO J, 2009, 28: 261-273

19 Johnsson N, Varshavsky A. Split ubiquitin as a sensor of protein interactions in vivo. Proc Natl Acad Sci USA, 1994, 91: 10340-10344

20 Zhang H, Xu Q, Stanislaw Krajewski, et al. BAR: an apoptosis regulator at the intersection of caspases and Bcl-2 family proteins . Proc Natl Acad Sci USA, 2000, 97: 2597-2602

21 Chu C C, Gao J P, Ho Y S. Over-expression of a modified bifunctional apoptosis regulator protects against cardiac injury and doxorubicin-induced cardiotoxicity in transgenic mice. Cardiovasc Res,
2009, 81: 20-27

22 Rong J, Chen L L, Toth J I, et al. Bifunctional apoptosis regulator (BAR), an endoplasmic reticulum (ER)-associated E3 ubiquitin ligase, modulates BI-1 protein stability and function in ER stress. J Biol Chem, 2011, 286: 1453-1463

23 Ye X, Mehlen P, Rabizadeh S, et al. TRAF family proteins interact with the common neurotrophin receptor and modulate apoptosis induction. J Biol Chem, 1999, 274: 30202-30208

24 Bai Y, Li Q, Yang J, et al. p75NTR activation of NF- $\kappa$ B is involved in PrP106-126-induced apoptosis in mouse neuroblastoma cells. Neurosci Res, 2008, 62: 9-14

25 Andrea C, Elisabetta P, Anton J H, et al. Neurotrophin p75 Receptor $\left(\mathrm{p} 75^{\mathrm{NTR}}\right)$ promotes endothelial cell apoptosis and inhibits angiogenesis: implications for diabetes-induced impaired neovascularization in ischemic limb muscles. Circ Res, 2008, 103: e15-e26

26 Küchler J, Hartmann W, Waha A, et al. p75(NTR) induces apoptosis in medulloblastoma cells. Int J Cancer, 2011, 128: 1804-1812

Open Access This article is distributed under the terms of the Creative Commons Attribution License which permits any use, distribution, and reproduction in any medium, provided the original author(s) and source are credited. 